\title{
Category-Selective Top-Down Modulation in the Fusiform Face Area of the Human Brain During Visual Search
}

\author{
Salman Ul Hassan Dar*†, Tolga Çukur* ${ }^{* \dagger}$ \\ *Electrical and Electronics Engineering Department, Bilkent University, Ankara, Turkey \\ ${ }^{\dagger}$ National Magnetic Resonance Research Center, Bilkent University, Ankara, Turkey \\ ${ }_{\ddagger}^{\ddagger}$ Neuroscience Program, Bilkent University, Ankara, Turkey \\ Email: \{salman, cukur\}@ee.bilkent.edu.tr
}

\begin{abstract}
Several regions in the ventral-temporal cortex of the human brain are thought to have representations of specific categories of objects. Furthermore, a distributed network of frontal and parietal brain regions is implicated in attentional control. It is assumed that during visual search, attention-control regions send top-down signals to the target category-selective areas to bias the processing in favour of the attended object category. However, little is known about such causal interactions during naturalistic visual search. Here we assess the influence of attention-control brain regions on a well-known face selective area fusiform face area (FFA) during natural visual search using Granger causality analysis. Our results indicate that attending to humans enhances the influence of attention-control regions on the fusiform face area.
\end{abstract}

Index Terms-Brain connectivity, category selectivity, Granger causality, functional MRI

\section{INTRODUCTION}

The human brain comprises various regions in the ventraltemporal cortex that are selective for ecologically important categories of objects like 'human faces' and 'body parts' [1], [2]. Furthermore, a distributed network of frontal and parietal regions (intraparietal sulcus (IPS), frontal eye fields (FEF), supplementary eye fields (SEF), frontal operculum (FO)) is shown to be involved in attentional control [3]-[5]. It is believed that when we search for an object category, the attention-control regions send top-down signals to the target category-selective regions to bias the processing in favour of the attended category. Few studies have shown such causal interactions between attention-control and category-selective areas during object-based attention [6]-[8]. These studies showed that while attending to an object, attention-control regions in frontal lobe increased their influence on the target category-selective areas. In these studies, human subjects were shown overlapping images of a small number of stimuli. Furthermore, these stimuli were presented at fixed locations. However, in real world we have to search for the targets in much more complex and cluttered scenes. In this study, we focused on causal effects of a broad network of attentioncontrol areas on a well-known face selective area (fusiform

978-1-5090-6494-6/17/\$31.00 2017 IEEE face area (FFA)) during real-world visual search [1], [9]. For this purpose, we used an experimental paradigm in which human subjects were shown natural movies [10]. In these natural movies, the subjects were instructed to search for 'humans' or 'vehicles' in distinct runs. If attention enhances the influence of attention-control regions on category-selective regions based on the target category, then the influence of attention-control regions on FFA should be stronger during human search, as compared to during vehicle search. To obtain the magnitude of influence of attention-control regions on FFA, we employed Granger causality analysis [11]. Granger causality uses the idea of temporal precedence. It exploits the fact that the cause precedes its effect. To examine if the causal interactions depend on the attended category, we compared the Granger causality measure across the visual search tasks.

\section{Methods}

\section{A. Experiment and Stimulus}

MRI data were acquired from five human subjects. We collected functional data using a $T_{2}^{*}$-weighted gradient-echo sequence. Contamination caused by the fat signal while acquiring MRI data is an important issue in neuroimaging experiments [12]-[15]. For this purpose, sequence with fat suppression was used for fMRI data acquisition. Anatomical data were acquired using a magnetization prepared 3D sequence [16]-[19]. For each subject head motion correction was performed by aligning all functional volumes to the first volume of the functional run [20].

The subjects were shown 30 minutes of identical natural movies twice. They were instructed to covertly search for humans or vehicles in separate runs. In addition, to ensure good behavioural performance, the subjects were given a response button, and they were instructed to press the response button on detecting the target category.

The natural movie stimulus was made by compiling 10-20 s short video clips. The clips were chosen and arranged in such a way that four types of stimuli (humans, vehicles, both humans and vehicles, none of them) appeared for an equal amount of time. 


\section{B. Granger Causality}

To examine the influence of attention-control areas on the face selective region FFA, Granger causality analysis was employed [11]. Consider time series of two brain regions $X$ and $Y$. Region $Y$ is said to Granger cause $X$, if the past values of the region $Y$ along with the past values of the region $X$ are better predictors of the present values of the region $X$, as compared to the past values of the region $X$ alone.

To obtain the Granger causality measure, first, the present values of the region $X$ are predicted using an autoregressive model. Autoregressive model uses linear combination of the past values of a variable to predict its present values.

$$
X(t)=\sum_{k=1}^{p} A_{x x}^{\prime}(k) \cdot X(t-k)+\varepsilon_{x}^{\prime}(t),
$$

where $A_{x x}^{\prime}$ quantifies the effect of $p$ past values on the present value of the region $X$, and $\varepsilon_{x}^{\prime}$ are the residuals. $A_{x x}^{\prime}$ was obtained using the least squares approach.

After obtaining the residuals from the autoregressive model, again similar procedure is repeated, however, this time the past values of $Y$ are also added as regressors in the autoregressive model.

$X(t)=\sum_{k=1}^{p} A_{x x}(k) \cdot X(t-k)+\sum_{k=1}^{p} A_{x y}(k) \cdot Y(t-k)+\varepsilon_{x}(t)$,

where $A_{x x}$ quantifies the effect of $p$ past values of both $X$ and $Y$ on the present value of the region $X$, and $\varepsilon_{x}$ are the residuals. The Granger causality measure from the region $Y$ to the region $X$ is afterwards obtained by using Granger causality index (GCI). GCI is given by:

$$
G C I_{Y \rightarrow X}=1-\frac{\operatorname{var}\left(\varepsilon_{x}(t)\right)}{\operatorname{var}\left(\varepsilon_{x}^{\prime}(t)\right)} .
$$

GCI is a normalized measure and lies in the range [0,1]. GCI was used to make the Granger causality measure across the attention conditions comparable.

Granger causality is obtained by computing ratio of the residual terms, $\operatorname{var}\left(\varepsilon_{x}(t)\right)$ and $\operatorname{var}\left(\varepsilon_{x}^{\prime}(t)\right)$. Therefore, increasing the value of $p$ decreases the ratio, and after a certain value of $p$, the model starts overfitting. To obtain the optimum value of $p$, Bayesian information criterion (BIC) was used [21]. BIC is given by:

$$
B I C=-2 \ln \hat{L}+k \cdot \ln (n),
$$

where $\hat{L}$ is the likelihood, that is obtained by predicting present values of both $X$ and $Y$ using past values of both $X$ and $Y . k$ is the number of previous time lags used to predict the present values of $X$, and $n$ is the total number of time points. The likelihood is given as:

$$
\hat{L}=-\frac{1}{n} \sum_{t=1}^{n}\left(Z(t)-\hat{Z(t))^{2}}\right.
$$

where $Z(t)$ represents the multivariate time series of regions $(X$ and $Y$ ) for which we are trying to estimate $p$, and $\hat{Z(t)}$

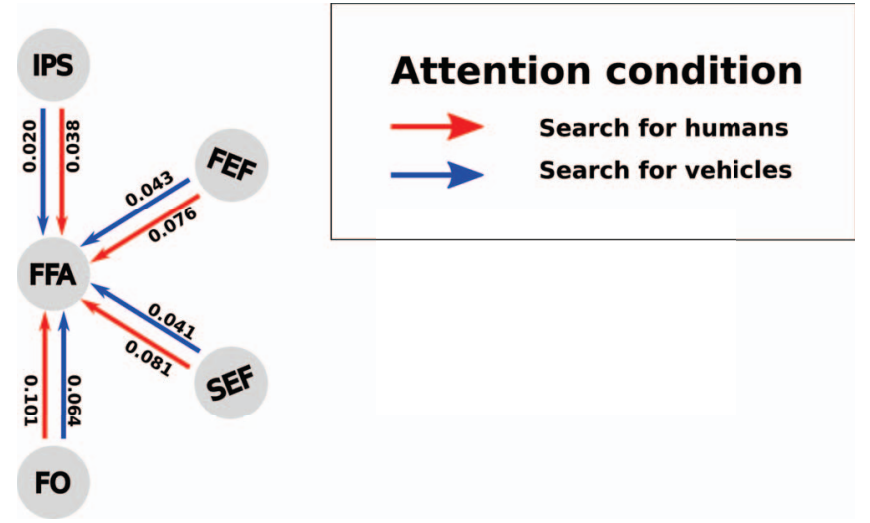

Fig. 1. Granger causality from attention-control regions (IPS, FEF, SEF, FO) to FFA. We computed the Granger causality measure from attention-control regions to FFA for both attention conditions, and averaged the measures across the subjects. Blue color shows causality while attending to vehicles, and red color shows causality while attending to humans.

represents the predicted values obtained by using the least squares approach. BIC is directly proportional to the negative $\log$-likelihood function and the number of previous time lags used to predict the present values. Therefore, the model with the lowest value of BIC is chosen to obtain Granger causality. We computed Granger causality from attention-control regions to FFA separately for both attention conditions. We then measured the percentage change in Granger causality between the two attention conditions.

We carried out the aforementioned analysis on conventional functional brain regions. To investigate if there are additional regions in the human brain that change their influence on FFA based on the target category, we repeated similar procedures. However instead of computing the causality from attentioncontrol to FFA, we obtained causality from all cortical voxels to FFA for both attention conditions. We measured the differences in causality across the attention conditions, and projected them onto flattened representation of the cortical surface.

\section{Bootstrap Test}

To examine if the percent changes in Granger causality were significant, they were passed through the bootstrap significance test. Bootstrap test is preferred over other statistical tests when the sample size is small. In our study, the sample consisted of five observations. Hence, we employed bootstrap test. This test uses bootstrap re-sampling method to generate resamples. In bootstrap, re-sampling is done with replacement. This means that in each re-sample, an observation can occur multiple times [22]. In this study, the sample size was five. Therefore, re-samples containing all possible combinations of observations $\left(5^{5}\right)$ were generated to obtain a distribution of the sample mean. Using the sample mean distribution, the pvalue was evaluated and the change in Granger causality was labelled as significant or in-significant. 


\section{RESULTS}

We computed Granger causality from attention-control regions to FFA for both attention conditions. We found that average magnitude of causality across the subjects was stronger during human search (Fig. 1). However, to examine if these differences were significant or not, they were passed through the bootstrap significance test (Fig. 2). We found that causality from all attention-control regions to FFA was significantly greater during human search, as compared to during vehicle search (Bootstrap test; $p<0.05$ ).

To investigate if there are additional brain regions that change their influence on FFA based on the target category, we obtained Granger causality from cortical voxels to FFA for both attention conditions. We measured the differences across the attention conditions and projected them onto cortical flatmaps (Fig. 3). As expected, we found that many voxels in the attention-control regions (IPS, FEF, SEF, FO) in frontal and parietal lobe increased their influence on FFA during human search. However, several voxels in the temporal parietal junction (TPJ) increased their influence on FFA during vehicle search, as compared to during human search. Temporal parietal junction is a part of ventral attention network, that is involved in re-orienting of attention to unattended stimuli [23]. Appearance of humans during vehicle search might have caused the increase in influence from TPJ to FFA to re-orient the attention to the unattended object category, i.e., humans during vehicle search.

\section{DISCUSSION}

Here, we used Granger causality to show that attentioncontrol regions increase their influence on category-selective areas, based on the target category. These results are in line

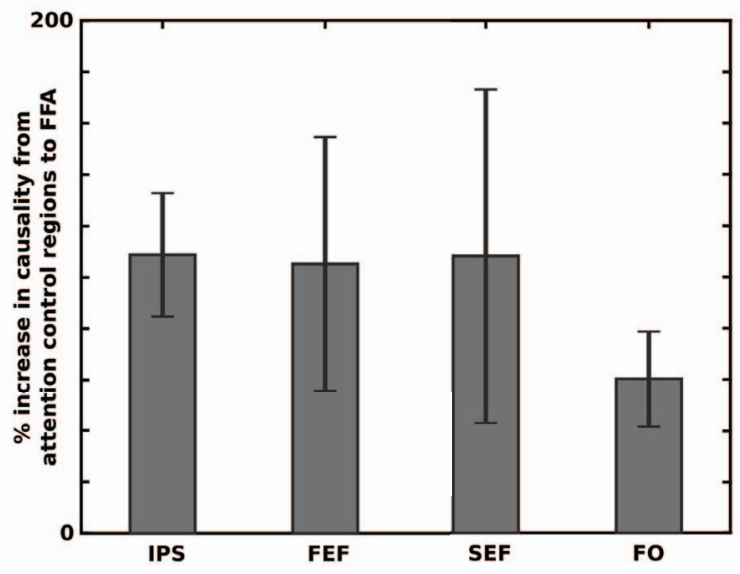

Fig. 2. Attentional increase in Granger causality during human search (\% changes \pm s.e.m). We computed the percentage differences of Granger causality between both attention conditions for all subjects. These differences were passed through the bootstrap significance test. The Granger causality measures from attention-control regions to FFA were significantly stronger while attending to humans, as compared to vehicles $(p<0.05)$.

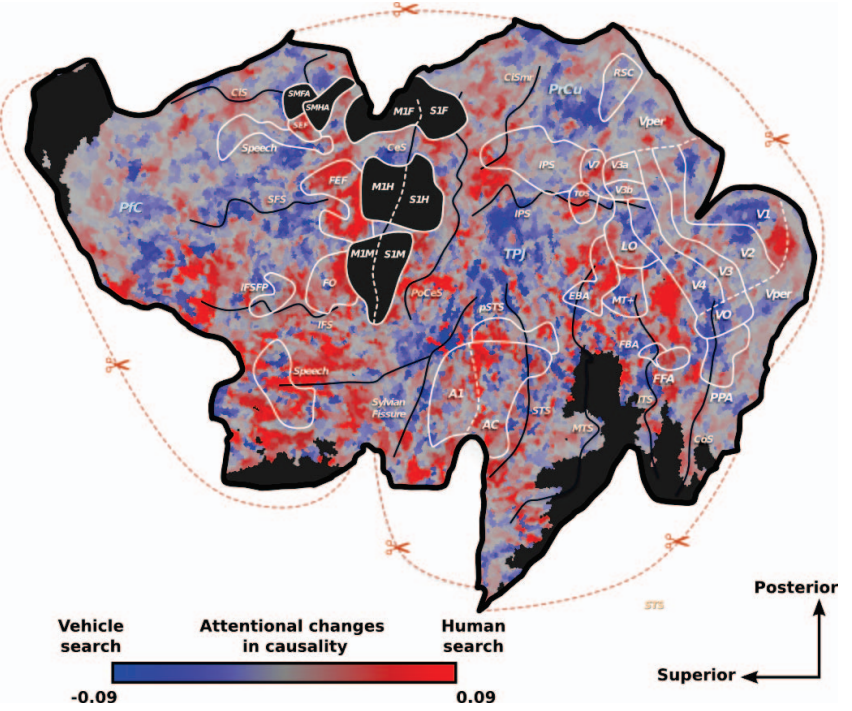

Fig. 3. Changes in Granger causality from cortical voxels to FFA, projected onto flattened representation of the cortical surface (Subject 1). The Granger causality measures from all cortical voxels to FFA were computed separately for both attention conditions. Red color represents stronger causality while attending to humans, and blue color represents stronger causality while attending to vehicles.

with the previous findings focusing on object-based attention [6]-[8]. However, previous studies focused on a much simpler stimulus. Here we used complex dynamic movies as a stimulus. Furthermore, previous findings were limited to a single attention-control area. However, we observed that a distributed network of attention-control regions was involved in attentional control.

Here our analysis was limited to FFA. It would be interesting to see how attention-control areas change their influence on other human-related category-selective areas like extrastriate body area (EBA), occipital face area (OFA), and superior temporal sulcus (STS) [2], [24], [25]. Furthermore, it has been shown that there are several areas in the human brain that are selective for vehicle-related object categories (parahippocampal place area (PPA), retrosplenial complex (RSC), and occipital place area (OPA)) [26]. We can also extend our study by carrying out similar analyses on the areas that are selective for vehicle-related categories.

Furthermore, Granger causality gives information about temporal precedence of one time course over the other. We can employ recently developed parallel imaging techniques to increase the temporal resolution of the acquired data [27][31].

We showed that a distributed network of attention-control areas is involved in attentional control. However, we cannot infer anything about the function of each attention-control region. Designing an experiment that studies function of each attention-control region separately can be an important aspect of the future work. Furthermore, Granger causality is an exploratory analysis. We can employ hypothesis driven techniques like dynamic causal modelling to test a specific 
hypothesis about the function of each attention-control region [32].

\section{ACKNOWLEDGMENT}

This work was supported in part by a European Molecular Biology Organization Installation Grant (IG 3028), a Marie Curie Actions Career Integration Grant (PCIG13-GA-2013618101), a TUBITAK 3501 Career Grant (114E546) and a TUBA GEBIP fellowship.

\section{REFERENCES}

[1] N. Kanwisher, J. McDermott, and M. M. Chun, "The fusiform face area: a module in human extrastriate cortex specialized for face perception," The Journal of Neuroscience, vol. 17, no. 11, pp. 4302-4311, 1997.

[2] P. E. Downing, Y. Jiang, M. Shuman, and N. Kanwisher, "A cortical area selective for visual processing of the human body," Science, vol. 293, no. 5539, pp. 2470-2473, 2001.

[3] N. Kanwisher and E. Wojciulik, "Visual attention: insights from brain imaging," Nature Reviews Neuroscience, vol. 1, no. 2, pp. 91-100, 2000.

[4] T. Higo, R. B. Mars, E. D. Boorman, E. R. Buch, and M. F. Rushworth, "Distributed and causal influence of frontal operculum in task control," Proceedings of the National Academy of Sciences, vol. 108, no. 10, pp. 4230-4235, 2011.

[5] M. Corbetta and G. L. Shulman, "Control of goal-directed and stimulusdriven attention in the brain," Nature reviews neuroscience, vol. 3 , no. 3 , pp. 201-215, 2002.

[6] D. Baldauf and R. Desimone, "Neural mechanisms of object-based attention," Science, vol. 344, no. 6182, pp. 424-427, 2014.

[7] K. Heinen, E. Feredoes, N. Weiskopf, C. C. Ruff, and J. Driver, "Direct evidence for attention-dependent influences of the frontal eye-fields on feature-responsive visual cortex," Cerebral Cortex, vol. 24, no. 11 , pp. 2815-2821, 2014.

[8] Y. Morishima, R. Akaishi, Y. Yamada, J. Okuda, K. Toma, and K. Sakai, "Task-specific signal transmission from prefrontal cortex in visual selective attention," Nature Neuroscience, vol. 12, no. 1, pp. 85-91, 2009

[9] T. Çukur, A. G. Huth, S. Nishimoto, and J. L. Gallant, "Functional subdomains within human ffa," Journal of Neuroscience, vol. 33, no. 42, pp. 16748-16766, 2013.

[10] T. Çukur, S. Nishimoto, A. G. Huth, and J. L. Gallant, "Attention during natural vision warps semantic representation across the human brain," Nature Neuroscience, vol. 16, no. 6, pp. 763-770, 2013.

[11] A. Roebroeck, E. Formisano, and R. Goebel, "Mapping directed influence over the brain using granger causality and fmri," Neuroimage, vol. 25, no. 1, pp. 230-242, 2005.

[12] T. Çukur and D. G. Nishimura, "Multiple repetition time balanced steady-state free precession imaging," Magnetic resonance in medicine, vol. 62, no. 1, pp. 193-204, 2009.

[13] B. Quist, B. A. Hargreaves, T. Cukur, G. R. Morrell, G. E. Gold, and N. K. Bangerter, "Simultaneous fat suppression and band reduction with large-angle multiple-acquisition balanced steady-state free precession," Magnetic resonance in medicine, vol. 67, no. 4, pp. 1004-1012, 2012.

[14] T. Çukur, N. K. Bangerter, and D. G. Nishimura, "Enhanced spectral shaping in steady-state free precession imaging," Magnetic resonance in medicine, vol. 58, no. 6, pp. 1216-1223, 2007.

[15] T. Çukur and D. G. Nishimura, "Fat-water separation with alternating repetition time balanced ssfp," Magnetic resonance in medicine, vol. 60 no. 2, pp. 479-484, 2008

[16] T. Çukur, J. H. Lee, N. K. Bangerter, B. A. Hargreaves, and D. G. Nishimura, "Non-contrast-enhanced flow-independent peripheral mr angiography with balanced ssfp," Magnetic resonance in medicine, vol. 61, no. 6 , pp. $1533-1539,2009$.

[17] N. K. Bangerter, T. Cukur, B. A. Hargreaves, B. S. Hu, J. H. Brittain, D. Park, G. E. Gold, and D. G. Nishimura, "Three-dimensional fluid-suppressed t2-prep flow-independent peripheral angiography using balanced ssfp," Magnetic resonance imaging, vol. 29, no. 8, pp. 11191124, 2011.

[18] K. T. Kwon, H. H. Wu, T. Shin, T. Çukur, M. Lustig, and D. G. Nishimura, "Three-dimensional magnetization-prepared imaging using a concentric cylinders trajectory," Magnetic resonance in medicine, vol. 71 , no. 5, pp. 1700-1710, 2014.
[19] T. Çukur, A. Shimakawa, H. Yu, B. A. Hargreaves, B. S. Hu, D. G. Nishimura, and J. H. Brittain, "Magnetization-prepared ideal bssfp: A flow-independent technique for noncontrast-enhanced peripheral angiography," Journal of Magnetic Resonance Imaging, vol. 33, no. 4, pp. 931939, 2011.

[20] M. Aksoy, C. Forman, M. Straka, T. Çukur, J. Hornegger, and R. Bammer, "Hybrid prospective and retrospective head motion correction to mitigate cross-calibration errors," Magnetic resonance in medicine, vol. 67 , no. 5, pp. 1237-1251, 2012 .

[21] E. Wit, E. v. d. Heuvel, and J.-W. Romeijn, "all models are wrong...: an introduction to model uncertainty," Statistica Neerlandica, vol. 66, no. 3, pp. 217-236, 2012

[22] C. H. Yu, "Resampling methods: concepts, applications, and justification," Practical Assessment, Research \& Evaluation, vol. 8, no. 19, pp. $1-23,2003$.

[23] M. Corbetta, G. Patel, and G. L. Shulman, "The reorienting system of the human brain: from environment to theory of mind," Neuron, vol. 58 , no. 3, pp. 306-324, 2008.

[24] I. Gauthier, M. J. Tarr, J. Moylan, P. Skudlarski, J. C. Gore, and A. W. Anderson, "The fusiform face area is part of a network that processes faces at the individual level," Journal of cognitive neuroscience, vol. 12, no. 3, pp. 495-504, 2000.

[25] A. D. Engell and J. V. Haxby, "Facial expression and gaze-direction in human superior temporal sulcus," Neuropsychologia, vol. 45, no. 14, pp. 3234-3241, 2007.

[26] T. Çukur, A. G. Huth, S. Nishimoto, and J. L. Gallant, "Functional subdomains within scene-selective cortex: Parahippocampal place area, retrosplenial complex, and occipital place area," Journal of Neuroscience, vol. 36, no. 40, pp. 10257-10273, 2016.

[27] T. Cukur, M. Lustig, and D. G. Nishimura, "Improving non-contrastenhanced steady-state free precession angiography with compressed sensing," Magnetic resonance in medicine, vol. 61, no. 5, pp. 1122 1131, 2009.

[28] T. Cukur, M. Lustig, E. U. Saritas, and D. G. Nishimura, "Signal compensation and compressed sensing for magnetization-prepared $\mathrm{mr}$ angiography," IEEE transactions on medical imaging, vol. 30, no. 5, pp. 1017-1027, 2011.

[29] T. Çukur, M. Lustig, and D. G. Nishimura, "Multiple-profile homogeneous image combination: Application to phase-cycled ssfp and multicoil imaging," Magnetic resonance in medicine, vol. 60 , no. 3 , pp. 732-738, 2008.

[30] T. Çukur, J. M. Santos, D. G. Nishimura, and J. M. Pauly, "Varying kernel-extent gridding reconstruction for undersampled variable-density spirals," Magnetic resonance in medicine, vol. 59, no. 1, pp. 196-201, 2008.

[31] T. Çukur, "Accelerated phase-cycled ssfp imaging with compressed sensing," IEEE transactions on medical imaging, vol. 34, no. 1, pp. 107$115,2015$.

[32] K. J. Friston, L. Harrison, and W. Penny, "Dynamic causal modelling," Neuroimage, vol. 19, no. 4, pp. 1273-1302, 2003. 